\title{
Cultural competency for clinicians: ChemSex and coinfection
}

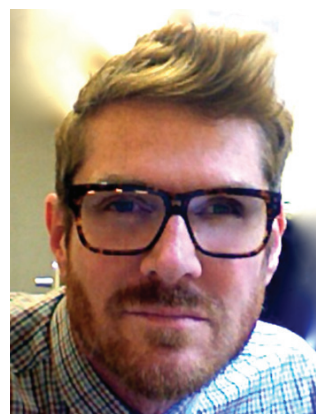

\begin{abstract}
David Stuart* speaks to Emily Hargrave, Commissioning Editor: David Stuart is the Substance Use Lead at London's 56 Dean Street (GUM/HIV services), addressing the sexualized drug use behaviors of MSM, commonly referred to as ChemSex. David has developed tailored interventions and care pathways for ChemSex trends, and has been instrumental in putting ChemSex on the Public Health agenda as well as pioneering the National Health Service and community responses.
\end{abstract}

Q Could you tell our readers a little about how your career began?

I had been volunteering with an opiate/crack cocaine service in London's Soho when ChemSex [1] trends among gay men (that is the sexualized drug used by gay men) began manifesting as a serious public health concern within that population. The lesbian, gay, bisexual and transgender (LGBT) project within that service was struggling to cope with increasing presentations of gay men reporting the use of crystal methamphetamine, mephedrone and GBL over 2-3-day 'sex benders' that were associated with multiple sexual partners, extreme or unsafe sexual practices, injecting drug use and sexually transmitted infections. In 2012, 90\% of the HIV/HCV-positive MSM clients visiting the service attributed their status' directly to the use of drugs and alcohol [2]. This early work enabled me to develop an understanding and familiarity with the context, motivations and epidemiology of these behaviors, and to begin developing care pathways and interventions to address this complicated syndemic. I was soon managing a targeted project for these trends at an LGBT charity, and in 2014, was employed by 56 Dean Street to address the trends within the genito-urinary medicine/HIV sector.

\section{Q What initially sparked your interest in working with those with HIV?}

Although I had been HIV positive myself since 1990, my interests had focused more on the cultural and holistic wellbeing of MSM drug users, and the underlying reasons driving the ChemSex syndemic. HIV was not the motivation for my work, just one of the many undesired consequences of ChemSex. I was struggling to get the issue on a public health agenda; the substance misuse sector felt this was a sexual health sector responsibility, and the sexual health sector felt it ought to be up to the substance misuse sector to respond. And while the buck was being passed backward and forth, more and more MSM were presenting for postexposure prophylaxis, or to their HIV appointments following ChemSex experiences. There are many gay men whose lives have been devastated, in many ways, by ChemSex, and by a myriad of consequences; but it is the HIV risks that secure its' place on the public health agenda. I felt very urgently that I ought to be doing my work right there, in the HIV prevention field.

*56 Dean Street, GUM/HIV, Chelsea \& Westminster hospital NHS Foundation Trust, London, UK;

David.stuart@chelwest.nhs.uk

\section{Vuture VIROGY}

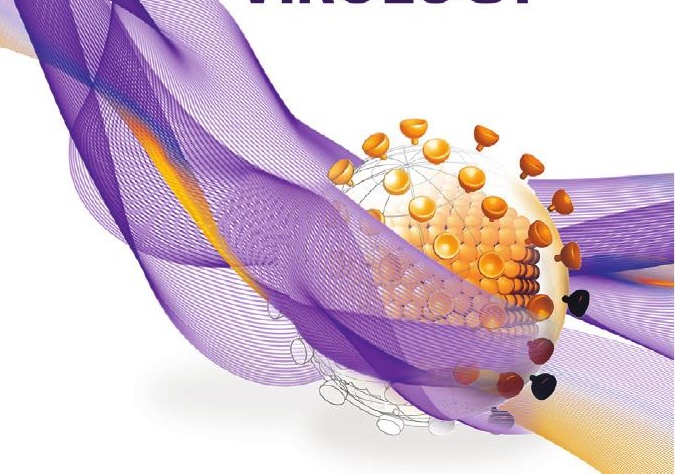


Q Have any colleagues, past or present, particularly influenced you \& your work? In the 1980s, Lord Norman Fowler battled to make needle and syringe provision available to injecting drug users from the public purse; it had been illegal to possess or dispense them prior to his battle, and many felt that giving out needles to 'junkies' was simply enabling selfindulgent drug users. Yet it became a primary tool to prevent the transmission of HIV among that population. ChemSex stimulates some similar responses; "Why don't those hedonistic, promiscuous gay men just stop doing drugs?”. Targeted ChemSex support ought to be provided as standard HIV prevention, without challenge or stigma, and funded by the public purse. It is simple HIV prevention. I identify with Lord Fowler's persistence to battle stigma, and find it inspiring.

\section{Q What is the most challenging part of your job?}

The most challenging is addressing the route causes of ChemSex. There have been challenges in putting it on the public health agenda, and developing tailored interventions; but that is just treating the problem. True prevention means addressing the stigmas that exist within and without gay communities about sex and disease, and understanding the full impact smartphone apps and technology are having on our sex and romantic lives. True prevention is incorporating LGBT-inclusive (age-appropriate) sex and relationship education in schools, so we may navigate the complicated world of modern sex, technology diversity and drug availability; and learn the role intimacy, respect and boundaries play in our sex/romantic lives.

\section{Q Additionally, what do you find to be the most rewarding?}

The most rewarding is undoubtedly the community engagement work. In 2014, Leigh Chislett and I began the Dean Street Wellbeing programme [3], a series of fun, creative and educational events that raise awareness, stimulate dialogue and address sexual and community wellbeing. The aim is to put sexual wellbeing at the heart of sexual health; if people experience good community cohesion, can robustly engage in dialogues on challenging topics, and experience good sexual/holistic wellbeing, good sexual health will follow. Less disease, less self-harm, less drug use and less poor mental health.
Q You have recently been involved with releasing a guide for clinicians \& health professionals surrounding HIV/HCV coinfection $\&$ substance abuse in men. How have these issues been addressed in the last 10 years? In substance misuse services, it has been well addressed; the risks of $\mathrm{HIV} / \mathrm{HCV}$ in the context of street homelessness/intravenous drug use have been well understood by staff in this field, and great work has been done to communicate safer practice to people at risk. The sexual transmission of HCV among MSM, however, has been marred by conflicting research about the transmission risks, and further marred by some clinicians not being fully aware of the specific contexts/details of certain gay sex practices, or in some cases, discomfort in discussing more extreme sexual practices with MSM patients.

\section{Q Why is this guide needed in current} healthcare?

ChemSex behaviors can be challenging, or difficult to comprehend for many of us; it can be extreme, appear self-harmful, illogical and yet our patients will continue to practice it despite our best interventions. There can be a great deal of shame associated with it for our patients, and so clinicians need a skill set of contextual and cultural competency and communication skills to effectively convey the risks to our patients. A 3-day, multiple-partner ChemSex environment is pregnant with the possibilities of $\mathrm{HIV} / \mathrm{HCV}$ infection, from many diverse sources; this guide [4] endeavors to equip healthcare providers with the language and contexts of such an environment to better inform our patients.

\section{Q Is HIV/HCV coinfection \& substance abuse} as much as a problem for women?

There is little evidence that injecting drug use by women is increasing in the United Kingdom; in fact it appears to be lessening, according to Public Health England [5]. That is not to say that we must not remain vigilant in asking about drug use in genito-urinary medicine/HIV services, and continuing to provide screening and support in drug services.
Q As a substance-abuse advisor, how do you see the adoption of this guide affecting the lives of patients who have HIV/HCV coinfection \& have sex when taking drugs? How will the guide help encourage testing \& treatment? 
Patients' shame and perceptions of judgement, as well as ignorance on behalf of clinicians are the greatest obstacles to HIV/HCV prevention among this population. This guide equips clinicians with colloquial terms and contextual detail that should facilitate more candid dialogue between ChemSex patients and clinicians. It should dispel any fears clinician's might have of appearing naïve of the behaviors, and it details the exact transmission risks in a ChemSex environment, hour by hour, play by play. A patient, who is convinced by a clinician's cultural awareness, will disclose more honestly his behaviors, and ask more boldly, questions relating to transmission. And a clinician can then more thoroughly equip his/her patient with accurate harm-reduction advice. This will significantly reduce infection rates and keep our patients confidently engaged in their healthcare.

\section{Q As someone who has devoted their career} to raising awareness and helping those with substance abuse, \& now in terms of HIV/HCV, what do you see as priorities for improving care \& advice further in this area in the UK? The priorities for me, continue to be improving the discourse between patients and their healthcare providers, as well as addressing stigma,

\section{References}

1 ChemSex; a working definition for healthcare providers.

www.chemsexsupport.com

2 Stuart D. Sexualised drug use by MSM: background, current status and response. ignorance and confusing messages regarding transmission, screening and treatment. The ChemSex syndemic is associated with chaotic drug use and extreme sexual behaviors, high rates of injecting drug use by an injecting-naive population among whom HIV and hepatitis C are highly prevalent. It is the perfect storm. Clinicians need a confident awareness of cultural context, language and risks, in order to effectively address this challenging public health concern.

\section{Disclaimer}

The opinions expressed in this interview are those of the interviewees and do not necessarily reflect the views of Future Medicine, Ltd.

\section{Financial \& competing interests disclosure}

$D$ Stuart has no relevant affiliations or financial involvement with any organization or entity with a financial interest in or financial conflict with the subject matter or materials discussed in the manuscript. This includes employment, consultancies, honoraria, stock ownership or options, expert testimony, grants or patents received or pending or royalties.

No writing assistance was utilized in the production of this manuscript. www.davidstuart.org/nursing-journal-1

3 www.deanstreetwellbeingprogramme.com

4 ChemSex and Hepatitis C. A Guide for Clinicians.

www.chemsexsupport.com
5 Data from National Drug Treatment Monitoring Service (NDTMS), Public Health England. www.gov.uk 